\title{
Defect topologies in a nematic liquid crystal near a patchy colloid
}

Cite as: J. Chem. Phys. 136, 194703 (2012); https://doi.org/10.1063/1.4717619

Submitted: 14 February 2012 - Accepted: 26 April 2012 • Published Online: 17 May 2012

Michael Melle, Sergej Schlotthauer, Marco G. Mazza, et al.

\section{ARTICLES YOU MAY BE INTERESTED IN}

Effects of flow on topological defects in a nematic liquid crystal near a colloid

The Journal of Chemical Physics 140, 054905 (2014); https://doi.org/10.1063/1.4862953

Defect topologies in chiral liquid crystals confined to mesoscopic channels

The Journal of Chemical Physics 142, 194704 (2015); https://doi.org/10.1063/1.4920979

Modeling flows of confined nematic liquid crystals

The Journal of Chemical Physics 134, 134905 (2011); https://doi.org/10.1063/1.3567098

The Journal

SPECIAL TOPIC: Low-Dimensional

of Chemical Physics Materials for Quantum Information Science 


\title{
Defect topologies in a nematic liquid crystal near a patchy colloid
}

\author{
Michael Melle, ${ }^{1}$ Sergej Schlotthauer, ${ }^{1}$ Marco G. Mazza, ${ }^{1}$ Sabine H. L. Klapp, ${ }^{2}$ \\ and Martin Schoen ${ }^{1,3, a)}$ \\ ${ }^{1}$ Stranski-Laboratorium für Physikalische und Theoretische Chemie, Fakultät für Mathematik und \\ Naturwissenschaften, Technische Universität Berlin, Straße des 17. Juni 135, 10623 Berlin, Germany \\ ${ }^{2}$ Institut für Theoretische Physik, Fakultät für Mathematik und Naturwissenschaften, Technische Universität \\ Berlin, Hardenbergstr. 36, 10623 Berlin, Germany \\ ${ }^{3}$ Department of Chemical and Biomolecular Engineering, Engineering Building I, Box 7905, North Carolina \\ State University, 911 Partners Way, Raleigh, North Carolina 27695, USA
}

(Received 14 February 2012; accepted 26 April 2012; published online 17 May 2012)

\begin{abstract}
Using isothermal-isobaric Monte Carlo simulations we investigate defect topologies due to a spherical colloidal particle immersed in a nematic liquid crystal. Defects arise because of the competition between the preferential orientation at the colloid's surface and the far-field director $\widehat{\boldsymbol{n}}_{0}$. Considering a chemically homogeneous colloid as a special case we observe the well-known surface and saturn ring defect topologies for weak and strong perpendicular anchoring, respectively; for homogeneous, strong parallel anchoring we find a boojum defect topology that has been seen experimentally [see P. Poulin and D. A. Weitz, Phys. Rev. E 57, 626 (1998)] but not in computer simulations. We also consider a heterogeneous, patchy colloid where the liquid-crystal molecules anchor either preferentially planar or perpendicular at the surface of the colloid. For a patchy colloid we observe a boojum ring defect topology in agreement with recent experimental studies [see M. Conradi, M. Ravnik, M. Bele, M. Zorko, S. Žumer, and I. Muševič, Soft Matter 5, 3905 (2009)]. We also observe two other novel defect topologies that have not been reported thus far neither experimentally nor theoretically.

(C) 2012 American Institute of Physics. [http://dx.doi.org/10.1063/1.4717619]
\end{abstract}

\section{INTRODUCTION}

Colloidal dispersions are ubiquitous in our everyday life. ${ }^{1}$ They appear in important applications such as food, cosmetics, ink, paints, or coatings. If these colloids are dispersed in rather simple carrier fluids the interactions between a pair of colloidal particles may have very different origins. ${ }^{1} \mathrm{Nev}-$ ertheless, many of these systems share a common feature in which the effective interactions (including the impact of the carrier fluid) between the colloidal particles have spherical symmetry. An example in this regard is a charge-stabilized solution (see, for example, Ref. 2). As a consequence, such colloids typically assemble into highly symmetric structures with cubic or hexagonal symmetry. ${ }^{3,4}$

In this context there is a particular interest in periodic assemblies of colloidal particles. For example, considering dielectric colloids as a special case it has been shown ${ }^{5}$ that the propagation of light is affected in a way similar to the propagation of electrons in a semiconductor crystal. Hence, periodic assemblies of colloids may be utilized to manufacture novel photonic devices with fascinating and unusual properties whose basic physics was explained already by Yablonovitch. ${ }^{6}$

In all these cases nematic liquid crystals have been utilized as carrier fluids. Typical for nematic systems is a high degree of (uniaxial) long-range orientational order combined with a lack of any but short-range positional order. One may envision physical situations in which the presence of colloidal particles changes the properties of their carrier fluids ${ }^{7}$ such

\footnotetext{
a)Electronic mail: martin.schoen@tu-berlin.de.
}

that long-range, anisotropic forces arise. These elastic forces are due to the distortion of the nematic director field. Such distortions are caused by the surface alignment of liquid-crystal molecules at the curved surface of a colloidal inclusion. On account of long-range elastic forces assemblies of quite complex and fascinating structures may form that would not exist in any simple carrier fluid. ${ }^{3,8-11}$ Immersing in such a nematic liquid crystal a colloidal particle may generate complex structures because the local director field $\widehat{\boldsymbol{n}}(\boldsymbol{r})$ in the vicinity of the colloid will generally differ from the far-field director $\widehat{\boldsymbol{n}}_{0}$.

The deviation between local and far-field directors is caused by the specific way liquid-crystal molecules (i.e., mesogens) are anchored at the surface of the colloid. "Anchoring" refers to an energetic discrimination of one or more molecular orientations of the mesogens at the surface of the colloid. Different anchoring scenarios can be realized by a number of different techniques ${ }^{12}$ down to nanometer-sized colloidal particles. ${ }^{13}$

As one moves away from the colloid's surface, $\widehat{\boldsymbol{n}}(\boldsymbol{r})$ has an increasingly stronger tendency to coincide eventually with $\widehat{\boldsymbol{n}}_{0}$. However, at specific points and characteristic distances from the colloid the remaining mismatch between $\widehat{\boldsymbol{n}}(\boldsymbol{r})$ and $\widehat{\boldsymbol{n}}_{0}$ causes topological defects. Defect topologies of colloidal dispersions in liquid-crystal carrier fluids have already received quite a bit of experimental interest. ${ }^{3,8-11,14}$

The defects may be thought of as topological charges that give rise to topologies that are either dipolar ${ }^{9-11}$ or quadrupolar in nature. ${ }^{14,15}$ By considering the interaction between dipole- and quadrupole-moment densities with $\widehat{\boldsymbol{n}}(\boldsymbol{r})$ one may develop a phenomenological theory capable of explaining the formation of assemblies of colloids due to a specific defect 
topology in the liquid crystal. For details of this approach we refer the interested reader to the monumental and lucid review by Stark. ${ }^{1}$

The above discussion already shows that the physics of colloids with chemically homogeneous surfaces immersed in a nematic liquid-crystal carrier fluid is very rich and fascinating. Even more interesting phenomena may therefore be anticipated if the colloid's surface is chemically heterogeneous. These colloids, usually referred to as "patchy" or "Janus" colloids, have been introduced quite some time ago by Casagrande and Veyssié. ${ }^{16}$ Since then the synthesis of Janus particles has become considerably more sophisticated. ${ }^{17}$ Nowadays it is feasible to control both size ${ }^{18}$ and patchiness ${ }^{19}$ of Janus colloids.

However, the interaction between patchy colloids and the director field of a nematic liquid crystal has received surprisingly little attention so far. Conradi et al. use a combination of optical microscopy and Landau-de Gennes theory to study the director field around Janus colloids. ${ }^{20}$ The Janus colloids are spherical and have been prepared such that one hemisphere anchors a mesogen planar whereas at the other hemisphere perpendicular anchoring dominates. The key result of this study is the observation of a novel defect topology that has not been reported before. In a later study Conradi et al. show that if Janus colloids are immersed in a liquid-crystal carrier fluid one may use laser light to switch irreversibly between different defect topologies. ${ }^{21}$

On the theoretical side it seems that defect topologies due to patchy colloids have only been investigated at meanfield level. ${ }^{20}$ Thus, in this work we are presenting a systematic Monte Carlo (MC) study of defect topologies caused by a single, immobilized patchy colloid. The remainder of our manuscript is organized as follows. In Sec. II we introduce our model system. Section III summarizes some key theoretical concepts relevant to this work. Our results are presented in Sec. IV and put into perspective in the concluding Sec. V.

\section{MODEL SYSTEM}

In this work we investigate the perturbation of the local director field of a nematic liquid crystal composed of $N$ mesogens that is caused by a single, spherical colloidal particle immobilized at the center of the computational cell. Fluid-fluid (ff) interactions between the liquid-crystal host molecules are described by the Hess-Su ${ }^{22}$ model potential given by

$$
\begin{aligned}
u_{\mathrm{ff}}\left(\boldsymbol{r}_{i j}, \widehat{\boldsymbol{u}}_{i}, \widehat{\boldsymbol{u}}_{j}\right)= & 4 \epsilon_{\mathrm{ff}}\left\{\left(\frac{\sigma}{r_{i j}}\right)^{12}-\left(\frac{\sigma}{r_{i j}}\right)^{6}\right. \\
& \left.\times\left[1+\Psi\left(\widehat{\boldsymbol{r}}_{i j}, \widehat{\boldsymbol{u}}_{i}, \widehat{\boldsymbol{u}}_{j}\right)\right]\right\},
\end{aligned}
$$

where $r_{i j}$ is the magnitude of the distance vector $\boldsymbol{r}_{i j}$ between centers of mass of mesogens $i$ and $j$ located at $\boldsymbol{r}_{i}$ and $\boldsymbol{r}_{j}$, respectively, and $\widehat{\boldsymbol{r}}_{i j} \equiv \boldsymbol{r}_{i j} / r_{i j}$. Unit vectors $\widehat{\boldsymbol{u}}_{i}$ and $\widehat{\boldsymbol{u}}_{j}$ specify the orientation of mesogens $i$ and $j$. Throughout this work notation ".. " indicates a unit vector. Hence, $u_{\mathrm{ff}}$ is a LennardJones potential where the attractive contribution is modified to account for different relative orientations of a pair of meso- gens. In Eq. (2.1), $\sigma$ denotes the "diameter" of a spherical reference molecule and $\epsilon_{\mathrm{ff}}$ is the depth of the attractive well in that reference model. The anisotropy of the fluid-fluid interaction is accounted for by the function

$$
\begin{aligned}
\Psi\left(\widehat{\boldsymbol{r}}_{i j}, \widehat{\boldsymbol{u}}_{i}, \widehat{\boldsymbol{u}}_{j}\right)= & 5 \varepsilon_{1} P_{2}\left(\widehat{\boldsymbol{u}}_{i} \cdot \widehat{\boldsymbol{u}}_{j}\right) \\
& +5 \varepsilon_{2}\left[P_{2}\left(\widehat{\boldsymbol{u}}_{i} \cdot \widehat{\boldsymbol{r}}_{i j}\right)+P_{2}\left(\widehat{\boldsymbol{u}}_{j} \cdot \widehat{\boldsymbol{r}}_{i j}\right)\right],
\end{aligned}
$$

where $2 \varepsilon_{1}=-\varepsilon_{2}=0.04$ are ansiotropy parameters. Equation (2.2) is obtained from a summation of certain Wigner matrices selected to preserve the head-tail symmetry of the molecules ${ }^{22}$ and to guarantee that

$$
\iint \mathrm{d} \widehat{\boldsymbol{u}}_{i} \mathrm{~d} \widehat{\mathrm{u}}_{j} \Psi\left(\widehat{\boldsymbol{r}}_{i j}, \widehat{\boldsymbol{u}}_{i}, \widehat{\boldsymbol{u}}_{j}\right)=0
$$

which holds because in Eq. (2.2)

$$
P_{2}(x) \equiv \frac{1}{2}\left(3 x^{2}-1\right)
$$

is the second Legendre polynomial. In other words, $\Psi$ remains unaltered if $\widehat{\boldsymbol{u}}_{i}$ and/or $\widehat{\boldsymbol{u}}_{j}$ are replaced by $-\widehat{\boldsymbol{u}}_{i}$ and/or $-\widehat{\boldsymbol{u}}_{j}$ (head-tail symmetry). Properties of the singlecomponent Hess-Su model have already been investigated in depth both in the bulk, ${ }^{23}$ at a single solid surface, ${ }^{24}$ and under nanoconfinement conditions..$^{25-28}$

The liquid crystal is confined between two planar, structureless solid substrates. The substrates are introduced as a means to fix the far-field director $\widehat{\boldsymbol{n}}_{0}$ (i.e., the positionindependent nematic director sufficiently far away from the colloid) in space. This approach was inspired by a typical experimental setup. ${ }^{14}$ Specifically, we take the interaction between a liquid-crystal molecule and the substrate to be described by the model potential

$$
u_{\mathrm{fs}}^{[k]}\left(z_{i}, \widehat{\boldsymbol{u}}_{i}\right)=\epsilon_{\mathrm{fs}}\left[\frac{2}{5}\left(\frac{\sigma}{z_{i} \pm s_{z} / 2}\right)^{10}-\left(\frac{\sigma}{z_{i} \pm s_{z} / 2}\right)^{4} g_{x}\left(\widehat{\boldsymbol{u}}_{i}\right)\right]
$$

assuming lower $(k=1)$ and upper substrates $(k=2)$ to be located at $-s_{z} / 2$ and $+s_{z} / 2$, respectively. We take the substrates to be separated by a distance $s_{z}=24 \sigma$ along the $z$-axis which is large enough to prevent the colloidal particle placed at the center of the simulation box from interacting directly with either one of the solid substrates. Throughout this work we take $\epsilon_{\mathrm{fs}} / \epsilon_{\mathrm{ff}}=5$. In Eq. (2.5),

$$
g_{x}\left(\widehat{\boldsymbol{u}}_{i}\right)=\left(\widehat{\boldsymbol{u}}_{i} \cdot \widehat{\boldsymbol{e}}_{x}\right)^{2}
$$

is the so-called anchoring function where $\widehat{\boldsymbol{e}}_{x}$ is a unit vector pointing along the (positive) $x$-axis of a Cartesian coordinate system. According to its definition, $0 \leq g_{x}\left(\widehat{\boldsymbol{u}}_{i}\right) \leq 1$ such that molecule $i$ receives an energy penalty if it is not perfectly aligned with the $x$-axis. This guarantees that in the nematic phase and in the absence of the spherical colloid, the far-field director $\widehat{\boldsymbol{n}}_{0}$ is pointing in the $x$-direction.

However, as we shall demonstrate below, the presence of a spherical colloid at the center of the computational cell will perturb $\widehat{\boldsymbol{n}}(\boldsymbol{r})$ in the vicinity of its surface. This is because the 
mesogens interact with the colloid via a Yukawa-like potential

$$
\begin{aligned}
u_{\mathrm{fc}}\left(\boldsymbol{r}_{i}, \widehat{\boldsymbol{u}}_{i}\right)= & \epsilon_{\mathrm{fc}}\left[a_{1}\left(\frac{\sigma}{r_{i}-r_{0}}\right)^{10}\right. \\
& \left.-a_{2} \frac{\exp \left[-\eta\left(r_{i}-r_{0}\right)\right]}{r_{i}-r_{0}} g\left(\widehat{\boldsymbol{r}}_{i}, \widehat{\boldsymbol{u}}_{i}\right)\right],
\end{aligned}
$$

where $r_{i}$ is the distance between the center-of-mass of mesogen $i$ and the center-of-mass of the colloid, $r_{0}$ is the hard-core radius of the colloid, and $\eta$ is a parameter screening the range of the attraction. Throughout this work we take $r_{0}=3 \sigma$ and consider $\eta \sigma=0.5$. Thus, the colloid is about 6 times larger in diameter than our nearly spherical mesogens. A similar size ratio was studied earlier by Andrienko et al. ${ }^{29}$ and by Grollau et al. ${ }^{30}$ In particular, the former authors varied $r_{0}$ between $3 \sigma$ and $15 \sigma$ and took the liquid crystal host fluid to consist of Gay-Berne molecules with an aspect ratio of 3. Parameters

$$
\begin{gathered}
a_{1}=\frac{1+\eta \sigma}{9-\eta \sigma}, \\
a_{2}=\frac{10 \exp (\eta \sigma)}{9-\eta \sigma}
\end{gathered}
$$

are introduced to guarantee that

$$
\left.\frac{\mathrm{d} u_{\mathrm{fc}}}{\mathrm{d} r_{i}}\right|_{r_{i}^{\min }}=0
$$

and that

$$
u_{\mathrm{fc}}\left(r_{i}^{\min }\right)=-\epsilon_{\mathrm{fc}}
$$

irrespective of $\eta$ where $r_{i}^{\min }=r_{0}+\sigma$ denotes the distance of the minimum of $u_{\mathrm{fc}}$ from the center-of-mass of the colloid. For all the cases studied here we take $\epsilon_{\mathrm{fc}} / \epsilon_{\mathrm{ff}}=4$.

Our main focus will be on the perturbation of $\widehat{\boldsymbol{n}}(\boldsymbol{r})$ by the presence of a patchy colloid consisting of surface regions that anchor a mesogen in different ways. As before different anchoring scenarios are realized via an anchoring function $g\left(\widehat{\boldsymbol{r}}_{i}, \widehat{\boldsymbol{u}}_{i}\right)$ introduced in Eq. (2.7). We consider a colloidal particle composed of patches that anchor a mesogen in either a parallel or a perpendicular fashion with respect to the surface of the colloid. This can be realized via

$$
g\left(\widehat{\boldsymbol{r}}_{i}, \widehat{\boldsymbol{u}}_{i}\right)=w_{\|}\left(\widehat{\boldsymbol{r}}_{i}\right) g_{\|}\left(\widehat{\boldsymbol{r}}_{i}, \widehat{\boldsymbol{u}}_{i}\right)+\alpha w_{\perp}\left(\widehat{\boldsymbol{r}}_{i}\right) g_{\perp}\left(\widehat{\boldsymbol{r}}_{i}, \widehat{\boldsymbol{u}}_{i}\right),
$$

where the dimensionless parameter $0 \leq \alpha \leq 1$ is introduced to weaken the perpendicular alignment relative to the parallel one. This is necessary because in the Hess-Su model molecules have a natural tendency to always orient themselves in a perpendicular fashion with respect to the surface of the colloid. In Eq. (2.11)

$$
\begin{gathered}
g_{\|}\left(\widehat{\boldsymbol{r}}_{i}, \widehat{\boldsymbol{u}}_{i}\right)=\left(1-\left|\widehat{\boldsymbol{u}}_{i} \cdot \widehat{\boldsymbol{r}}_{i}\right|\right)^{2}, \\
g_{\perp}\left(\widehat{\boldsymbol{r}}_{i}, \widehat{\boldsymbol{u}}_{i}\right)=\left(\widehat{\boldsymbol{u}}_{i} \cdot \widehat{\boldsymbol{r}}_{i}\right)^{2},
\end{gathered}
$$

where $\widehat{\boldsymbol{r}}_{i}$ points from the colloid's center-of-mass outward to the center-of-mass of mesogen $i$. As can be seen from
Eq. (2.11) both contributions to the overall anchoring function are weighted according to

$$
\begin{gathered}
w_{\|}\left(\widehat{\boldsymbol{r}}_{i}\right)=1-\gamma_{\|}\left(1-\widehat{\boldsymbol{r}}_{i} \cdot \widehat{\boldsymbol{e}}_{x}\right)^{\delta}, \\
w_{\perp}\left(\widehat{\boldsymbol{r}}_{i}\right)=1-\gamma_{\perp}\left(1+\widehat{\boldsymbol{r}}_{i} \cdot \widehat{\boldsymbol{e}}_{x}\right)^{2} .
\end{gathered}
$$

The weighting function $w_{\|}\left(\widehat{\boldsymbol{r}}_{i}\right)$ assumes its maximum value at the north pole of the colloid defined by $\widehat{\boldsymbol{r}}_{i} \cdot \widehat{\boldsymbol{e}}_{x}=+1$, whereas $w_{\perp}\left(\widehat{\boldsymbol{r}}_{i}\right)$ is largest at the south pole where $\widehat{\boldsymbol{r}}_{i} \cdot \widehat{\boldsymbol{e}}_{x}=-1$. In other words, the outward normal at the north pole points along the positive $x$-axis. Throughout this work we keep this orientation of the colloid fixed, again inspired by experimental situations where such a fixed orientation can be realized in a number of different ways. ${ }^{20,31,32}$ Because meaningful weighting functions have to be positive semidefinite, $\gamma_{\|} \leq 2^{-\delta}$ and $\gamma_{\perp} \leq \frac{1}{4}$. Both parameters control the sharpness of the transition between the different patches on the colloid, whereas the exponents $\delta$ and 2 in Eqs. (2.13) determine their size.

Plots in Fig. 1 illustrate the anisotropy of the $u_{\mathrm{fc}}\left(\boldsymbol{r}_{i}, \widehat{\boldsymbol{u}}_{i}\right)$ for two distinguished orientations of a mesogen and for a given set of parameters $\eta, \delta, \gamma_{\|}$, and $\gamma_{\perp}$. The plots show that the potential is most attractive if the orientation of the molecule matches exactly the alignment enforced by a given patch as one would have anticipated. If, on the other hand, a molecule is completely misaligned with respect to a specific patch the attraction between that molecule and the colloid is much weaker. Notice also that in Fig. 1 the transition from planar to perpendicular anchoring is rather smooth. Again, this feature resembles realistic experimental situations (see, for example, Fig. 1 of Ref. 20).

Our model is also capable of describing the interaction between a mesogen and a homogeneous colloid, that is, irrespective of $\widehat{\boldsymbol{r}}_{i}$ the anchoring is the same everywhere at the colloid's surface. For example, parallel anchoring can be realized by setting $w_{\|}\left(\widehat{\boldsymbol{r}}_{i}\right)=1$ and $w_{\perp}\left(\widehat{\boldsymbol{r}}_{i}\right)=0$. In a similar fashion we can realize a homogeneous colloid with perpendicular anchoring.

\section{THEORETICAL BACKGROUND}

\section{A. Statistical thermodynamics}

We now turn to a brief introduction of the isothermalisobaric ensemble on which the MC simulations of this study are based. For the model introduced in Sec. II, Gibbs' fundamental relation may be cast as

$$
\mathrm{d} \mathcal{U}=T \mathrm{~d} \mathcal{S}+\mu \mathrm{d} N+\mathrm{d} W_{\text {mech }}
$$

where $\mathcal{U}$ denotes internal energy, $T$ is the temperature, $\mathcal{S}$ is the entropy, $\mu$ is the chemical potential, and $N$ is the number of molecules. For the present system the mechanical work contribution may be cast as

$$
\mathrm{d} W_{\text {mech }}=-\boldsymbol{A}^{\mathrm{T}} \mathbf{P} \mathrm{d} \boldsymbol{s},
$$

where elements of the vectors $\boldsymbol{A}^{\mathrm{T}}=\left(A_{x}, A_{y}, A_{z}\right)$ and $\boldsymbol{s}^{\mathrm{T}}$ $=\left(s_{x}, s_{y}, s_{z}\right)$ are the $\alpha$-directed faces and side lengths of a rectangular volume accommodating our model system and $\mathbf{P}$ 

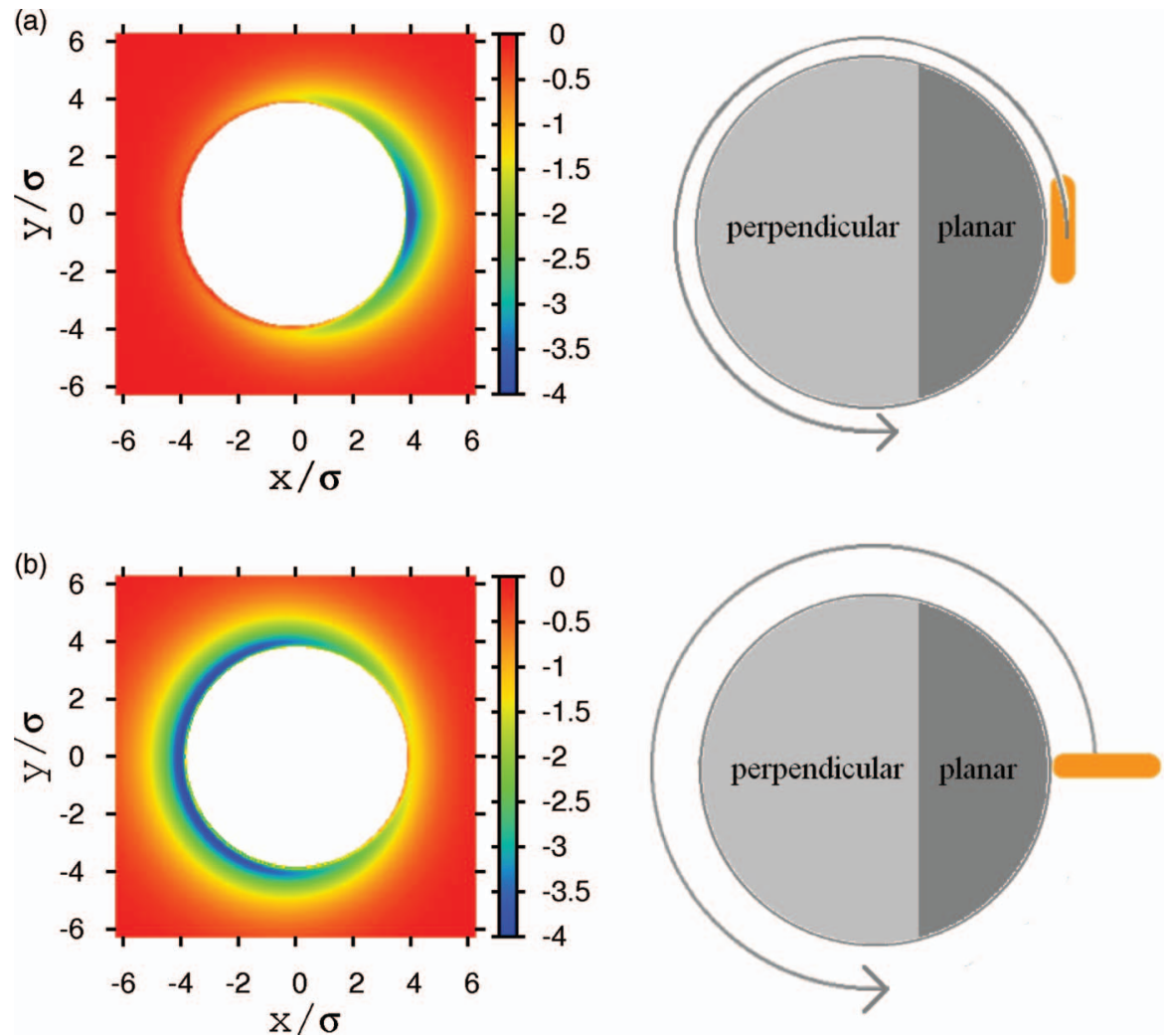

FIG. 1. Contour plots of $u_{\mathrm{fc}}(\boldsymbol{r}, \widehat{\boldsymbol{u}})$ (left) generated by moving a mesogen in the $x-y$ plane with fixed orientation relative to the local surface normal to the colloid (right) such that $\left|\widehat{\boldsymbol{u}}_{i} \cdot \widehat{\boldsymbol{r}}_{i}\right|=0$ (a) and $\left|\widehat{\boldsymbol{u}}_{i} \cdot \widehat{\boldsymbol{r}}_{i}\right|=1$ (b) [see Eqs. (2.11)-(2.13)]. The white area at the center of the contour plots is defined by the condition $u_{\mathrm{fc}}$ $\geq 0$; in colored parts, $u_{\mathrm{fc}}<0$ where the color (see attached color bar) indicates the local value of $u_{\mathrm{fc}}$ in units of $\epsilon_{\mathrm{ff}}$. Contour plots have been generated for $r_{0}$ $=3.0, \alpha=1.0, \eta=0.5, \delta=0.5, \gamma_{\|}=0.60, \gamma_{\perp}=0.15$, and $\epsilon_{\mathrm{fc}} / \epsilon_{\mathrm{ff}}=4.0$ [see Eqs. (2.7), (2.11), and (2.13)]. For illustrative purposes the anisotropy of the mesogen shown in the cartoons on the right is greatly exaggerated. In these cartoons areas shaded in darker and lighter gray indicate regions where the overall anchoring function $g(\widehat{\boldsymbol{u}})$ is dominated by $g_{\|}(\widehat{\boldsymbol{u}})$ and $g_{\perp}(\widehat{\boldsymbol{u}})$, respectively.

is the pressure tensor which can be represented by the $3 \times 3$ matrix

$$
\mathbf{P}=\left(\begin{array}{ccc}
P_{x x} & 0 & 0 \\
0 & P_{y y} & 0 \\
0 & 0 & P_{z z}
\end{array}\right)
$$

in the absence of shear strains. Because of the presence of the solid substrates and the (inhomogeneous) colloid, $P_{x x} \neq P_{y y}$ $\neq P_{z z}$ in general.

This is a situation analogous to the one considered by Diestler et $_{\text {al. }}{ }^{33}$ in their treatment of a fluid confined to a slitpore with atomically structured substrate surfaces. In this case the inequality of the two transverse components of $\mathbf{P}$, that is between $P_{x x}$ and $P_{y y}$, arises because the discrete atoms forming the substrate give rise to an inhomogeneous external field varying periodically in the $x-y$ plane; on account of the mere presence of a substrate, $P_{z z}$ generally differs from the transverse components. We therefore follow these earlier authors and rewrite Eq. (3.2) as

$$
\mathrm{d} W_{\text {mech }}=\mathcal{P}_{+} \mathrm{d} A+\mathcal{P}_{-} A \mathrm{~d} R-P_{z z} A \mathrm{~d} s_{z},
$$

where $A=A_{z}=s_{x} s_{y}, R=s_{x} / s_{y}$, and the interfacial tensions $\mathcal{P}_{+}$and $\mathcal{P}_{-}$are defined as

$$
\begin{aligned}
\mathcal{P}_{+} & =-\frac{s_{z}}{2}\left(P_{x x}+P_{y y}\right) \\
& \equiv-s_{z} P_{\|}
\end{aligned}
$$

$$
A \mathcal{P}_{-}=-\frac{s_{z}}{2 R}\left(P_{x x}-P_{y y}\right)
$$

With this choice of $A$ and $R$ as new state variables the contributions to $\mathrm{d} W_{\text {mech }}$ in Eq. (3.4) have a lucid interpretation. For example, the first term on the right side of Eq. (3.4) is the mechanical work associated with an affine transformation that changes the $z$-directed area of the system, whereas the second term represents the mechanical work associated with a change in the shape of the system while maintaining that area.

Next we introduce the Gibbs energy $\mathcal{G}$ through the Legendre transform $\mathrm{d} \mathcal{G}=\mathrm{d}\left(\mathcal{U}-T \mathcal{S}-\mathcal{P}_{+} A\right)$ such that

$$
\mathrm{d} \mathcal{G}=-\mathcal{S} \mathrm{d} T+\mu \mathrm{d} N-A \mathrm{~d} \mathcal{P}_{+}+\mathcal{P}_{-} A \mathrm{~d} R-P_{z z} A \mathrm{~d} s_{z}
$$

From Eq. (3.6) it is clear that $\mathcal{G}$ depends on the set $\left\{T, N, \mathcal{P}_{+}, R, s_{z}\right\}$ of natural variables. Thus, for any given set of values of those variables, $\mathcal{G}$ assumes a minimum in thermodynamic equilibrium.

To make contact with a molecular level of description we employ the expression ${ }^{34}$

$$
\mathcal{G}=-\beta^{-1} \ln \chi,
$$


where $\beta \equiv 1 / k_{\mathrm{B}} T, \quad k_{\mathrm{B}}$ is Boltzmann's constant, and $\chi\left(T, N, \mathcal{P}_{+}, R, s_{z}\right)$ is the partition function given by

$$
\chi=\sum_{A} \exp \left(-\beta \mathcal{P}_{+} A\right) \mathcal{Q}
$$

following, in principle, the detailed derivation presented in the book by Schoen and Klapp where $\mathcal{Q}$ is the canonical ensemble partition function in the classical limit. ${ }^{35}$ Equation (3.8) is the key expression from which all equilibrium properties of the carrier fluid may be derived. However, from the standpoint of an equilibrium computer simulation technique such as $\mathrm{MC}$, Eq. (3.8) is not immediately useful. This is because $\mathcal{Q}$ involves both kinetic and configurational contributions. For systems at thermodynamic equilibrium the former are irrelevant. One would therefore like to factorize $\mathcal{Q}$ into translational and rotational kinetic contributions and the configuration integral. According to the discussion presented by Gray and Gubbins ${ }^{36}$ and specialized later to molecular fluids with only two rotational degrees of freedom by Gruhn and Schoen ${ }^{37}$ such a factorization is not immediately possible but requires a suitable choice of variables. However, with such a choice $\mathcal{Q}$ may be written as

$$
\mathcal{Q}=\mathcal{Q}_{\mathrm{t}} \mathcal{Q}_{\mathrm{r}} \mathcal{Z}
$$

where the translational and rotational kinetic contributions are given by

$$
\begin{aligned}
& \mathcal{Q}_{\mathrm{t}}=\left(\frac{2 \pi m}{\beta h^{2}}\right)^{3 N / 2} \equiv \frac{1}{\Lambda^{3 N}}, \\
& \mathcal{Q}_{\mathrm{r}}=\frac{1}{\Lambda^{2 N}}\left(\frac{\mathfrak{I}}{m}\right)^{N},
\end{aligned}
$$

respectively. In Eqs. (3.10) and (3.11), $h$ is Planck's constant, $m$ is the mass of a mesogen, $\mathfrak{I}$ is its moment of inertia, and $\Lambda$ is the thermal de Broglie wavelength. The expressions for $\mathcal{Q}_{\mathrm{t}}$ and $\mathcal{Q}_{\mathrm{r}}$ are obtained by an analytic integration over linear and angular momentum subspaces, respectively.

The third term on the right side of Eq. (3.9)

$$
\mathcal{Z}=\frac{1}{2^{N}} \iint \mathrm{d} \boldsymbol{R} \mathrm{d} \widehat{\boldsymbol{U}} \exp [-\beta U(\boldsymbol{R}, \widehat{\boldsymbol{U}})]
$$

is the configuration integral. For notational convenience we have introduced shorthand notations $\boldsymbol{R}=\left\{\boldsymbol{r}_{1}, \boldsymbol{r}_{2}, \ldots, \boldsymbol{r}_{N}\right\}$, $\widehat{\boldsymbol{U}}=\left\{\widehat{\boldsymbol{u}}_{1}, \widehat{\boldsymbol{u}}_{2}, \ldots, \widehat{\boldsymbol{u}}_{N}\right\}, \mathrm{d} \boldsymbol{R}=\mathrm{d} \boldsymbol{r}_{1} \mathrm{~d} \boldsymbol{r}_{2} \ldots \mathrm{d} \boldsymbol{r}_{N}$, and, finally, $\mathrm{d} \widehat{\boldsymbol{U}}=\mathrm{d} \widehat{\boldsymbol{u}}_{1} \mathrm{~d} \widehat{\boldsymbol{u}}_{2} \ldots \mathrm{d} \widehat{\boldsymbol{u}}_{N}$. The prefactor $1 / 2^{N}$ corrects for double-counting equivalent configurations characterized by $\widehat{\boldsymbol{u}}_{i}$ and $-\widehat{\boldsymbol{u}}_{i}$ that arise on account of the head-tail symmetry of the mesogens (see Sec. II). In Eq. (3.12)

$$
\begin{aligned}
U(\boldsymbol{R}, \widehat{\boldsymbol{U}})= & \frac{1}{2} \sum_{i=1}^{N} \sum_{\substack{j=1 \\
i \neq j}}^{N} u_{\mathrm{ff}}\left(\boldsymbol{r}_{i j}, \widehat{\boldsymbol{u}}_{i}, \widehat{\boldsymbol{u}}_{j}\right) \\
& +\sum_{i=1}^{N} u_{\mathrm{fs}}^{[k]}\left(z_{i}, \widehat{\boldsymbol{u}}_{i}\right)+\sum_{i=1}^{N} u_{\mathrm{fc}}\left(r_{i}, \widehat{\boldsymbol{u}}_{i}\right)
\end{aligned}
$$

is the total configurational potential energy where we assume the interactions between the mesogens to be pairwise additive. The second and third terms on the right side of
Eq. (3.13) are sums of one-body contributions indicating that both the planar solid substrates and the colloid may be perceived as external fields exerted on the mesogens. Finally, from Eqs. (3.8)-(3.12) we have

$$
\chi=\frac{1}{\Lambda^{5 N}}\left(\frac{\mathfrak{I}}{m}\right)^{N} \sum_{A} \exp \left(-\beta \mathcal{P}_{+} A\right) \mathcal{Z}
$$

which is the desired expression because kinetic-energy contributions arise as rather trivial prefactors of the sum on $A$.

\section{B. Properties}

Because of the presence of the colloidal particle the liquid crystal in its vicinity will be inhomogeneous and anisotropic. In practice and as long as we are interested in effects in the proximity of the colloid we may employ the special symmetry of our system. To that end we realize that the line connecting the colloids north and south poles coincides with $\widehat{\boldsymbol{e}}_{x}$. Therefore, we have rotational symmetry around that axis and may project $\boldsymbol{r}$ onto the $x-z$ plane restricting ourselves to $z \geq 0$. For a molecule located at $\boldsymbol{r}$ the equivalent point $\boldsymbol{s}$ in the $x-z$ plane may be found through a rotation

$$
\boldsymbol{s}=\left(\begin{array}{ccc}
1 & 0 & 0 \\
0 & \cos \vartheta & -\sin \vartheta \\
0 & \sin \vartheta & \cos \vartheta
\end{array}\right) \boldsymbol{r}
$$

where $\vartheta=\operatorname{sgn}(y) \arccos \left(z / \sqrt{y^{2}+z^{2}}\right)$ is the angle between $\boldsymbol{r}$ and $s$. Because of this transformation all local quantities may be perceived as a function of $x$ and $z$.

Our main focus in this study is the deformation of the local director field $\widehat{\boldsymbol{n}}(\boldsymbol{r})$ in the nematic phase of the liquid crystal caused by the presence of the patchy colloid. To compute $\widehat{\boldsymbol{n}}(\boldsymbol{r})$ a convenient point of departure is the local alignment tensor ${ }^{38}$ (see also Ref. 39) which we define as

$$
\mathbf{Q}(\boldsymbol{r}) \equiv \frac{1}{2 \rho(\boldsymbol{r})} \sum_{i=1}^{N}\left\langle\left[3 \widehat{\boldsymbol{u}}_{i}\left(\boldsymbol{r}_{i}\right) \otimes \widehat{\boldsymbol{u}}_{i}\left(\boldsymbol{r}_{i}\right)-\mathbf{1}\right] \delta\left(\boldsymbol{r}-\boldsymbol{r}_{i}\right)\right\rangle
$$

where the operator " $\otimes$ " denotes the tensor (i.e., "dyadic") product and $\mathbf{1}$ is the unit tensor. Hence, $\mathbf{Q}(\boldsymbol{r})$ is a real, symmetric, traceless, second-rank tensor that can be represented by a $3 \times 3$ matrix. Using Jacobi's method the three eigenvalues $\lambda_{-}(\boldsymbol{r})<\lambda_{0}(\boldsymbol{r})<\lambda_{+}(\boldsymbol{r})$ may be obtained numerically. ${ }^{40}$ We take $\lambda_{+}(\boldsymbol{r})$ as the local nematic order parameter and the associated eigenvector $\widehat{\boldsymbol{n}}(\boldsymbol{r})$ as the local director. Again, the local nematic order parameter and the local director may be parametrized via $x$ and $z$.

This procedure follows that proposed earlier by Andrienko et al. in their study of topological defects in a liquid crystal caused by the presence of a homogeneous colloidal particle. ${ }^{29}$ Notice, however, that the computation of eigenvalues and eigenvectors is based upon an already ensemble averaged $\mathbf{Q}(\boldsymbol{r})$, whereas conventionally ${ }^{27,39,41-43}$ the instantaneous alignment tensor is used to compute its eigenvalues which are then ensemble-averaged subsequently. However, for reasons explained in Ref. 24 this approach could cause a substantial system size dependence of the local nematic order 
(a)

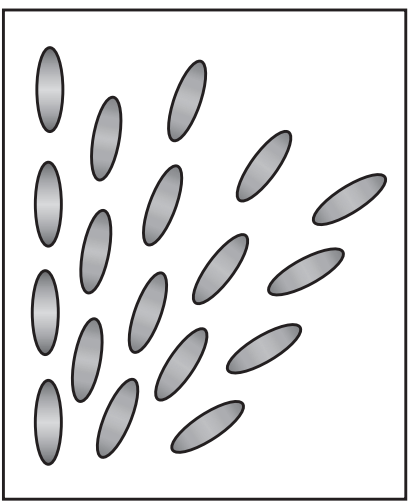

(b)

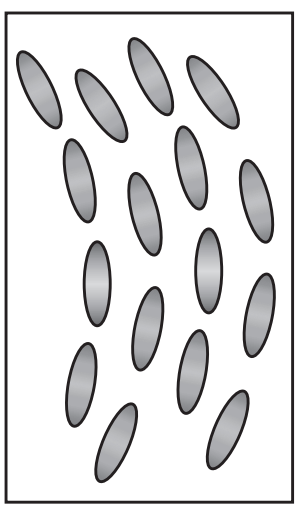

(c)

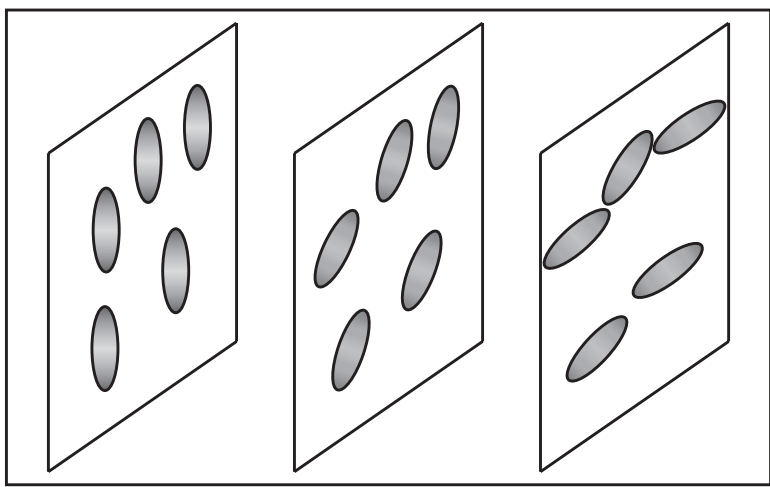

FIG. 2. Schematic representation of local splay (a), bend (b), and twist (c) configurations of a director field.

parameter that needs to be corrected for. The present approach avoids that problem by taking the ensemble average first and then diagonalizing $\mathbf{Q}(\boldsymbol{r})$ afterwards.

Another useful quantity to analyze is the so-called Frank free-energy density ${ }^{44}$ which in the one-constant approximation may be cast as

$$
\begin{aligned}
\mathcal{F}(\boldsymbol{r})= & \frac{K}{2}\left\{[\nabla \cdot \widehat{\boldsymbol{n}}(\boldsymbol{r})]^{2}+[\widehat{\boldsymbol{n}}(\boldsymbol{r}) \cdot \nabla \times \widehat{\boldsymbol{n}}(\boldsymbol{r})]^{2}\right. \\
& \left.+[\widehat{\boldsymbol{n}}(\boldsymbol{r}) \times \nabla \times \widehat{\boldsymbol{n}}(\boldsymbol{r})]^{2}\right\} .
\end{aligned}
$$

In Eq. (3.17), $K$ is a material constant and the three terms of the right side refer to contributions arising from splay, twist, and bend deformations of the director field (see Fig. 2). Notice that in principle a different elastic constant would be associated with each of the three terms on the right side of Eq. (3.17). We have adopted the one-constant approximation for simplicity and because we want to relate a given defect topology to one (or more) of the well understood deformations of $\widehat{\boldsymbol{n}}(\boldsymbol{r})$ (see also Sec. V). The magnitude of these three contributions is considered separately in Sec. IV D where we analyze them for a given defect topology observed for patchy colloids. We also notice in passing that minimizing the Frank free-energy density has been employed as a means to determine the equilibrium director field in the presence of a colloidal particle. ${ }^{45-47}$ In any event, a calculation of $\mathcal{F}(\boldsymbol{r})$ involves differentiation of the local director field with respect to position. We then computed $\nabla \times \widehat{\boldsymbol{n}}(\boldsymbol{r})$ and $\nabla \cdot \widehat{\boldsymbol{n}}(\boldsymbol{r})$ numerically by applying a standard finite-difference scheme to $\widehat{\boldsymbol{n}}(\boldsymbol{r})$.

However, in practice, a small technical problem arises due to the fact that $\widehat{\boldsymbol{n}}(\boldsymbol{r})$ and $-\widehat{\boldsymbol{n}}(\boldsymbol{r})$ are equivalent. This reflects the uniaxial symmetry of our molecules and is a con- sequence of the mathematical structure of $\mathbf{Q}(\boldsymbol{r})$. Hence, in a straightforward calculation of $\mathcal{F}(\boldsymbol{r})$ one runs into problems with the numerical finite-difference scheme if suddenly the local director changes sign. Therefore, one needs to make sure that neighboring local directors are always pointing into the same direction. In practice, this is achieved by the following approach. Suppose, $\widehat{\boldsymbol{n}}\left(\boldsymbol{r}_{0}\right)$ is the local nematic director at some reference point $\boldsymbol{r}_{0}$. If the scalar product between $\widehat{\boldsymbol{n}}\left(\boldsymbol{r}_{0}\right)$ and a local director $\widehat{\boldsymbol{n}}(\boldsymbol{r})$ at another point $\boldsymbol{r}$ in the immediate vicinity of $\boldsymbol{r}_{0}$ is negative, $\widehat{\boldsymbol{n}}(\boldsymbol{r})$ is replaced by $-\widehat{\boldsymbol{n}}(\boldsymbol{r})$ such that during the numerical differentiation discontinuous changes are avoided. Notice that this change in sign prior to the numerical differentiation of $\widehat{\boldsymbol{n}}(\boldsymbol{r})$ does not affect the final result for $\mathcal{F}(\boldsymbol{r})$ which depends quadratically on the local derivatives of $\widehat{\boldsymbol{n}}(\boldsymbol{r})$.

\section{RESULTS}

\section{A. Numerical details}

The MC simulations on which this study is based have been performed in a specialized isothermal-isobaric ensemble where the thermodynamic state is specified by fixed values of $N, T, R, s_{\mathrm{z}}$, and $P_{\|}$[see Eq. (3.5a)].

Employing a conventional Metropolis algorithm adapted to simulations in the specialized isothermal-isobaric ensemble we are generating a Markov chain with a limiting distribution in configuration space proportional to $\exp \left\{-\beta\left[U(\boldsymbol{R}, \widehat{\boldsymbol{U}})+P_{+} A-N \beta^{-1} \ln A\right]\right\}$. To achieve this goal the adapted Metropolis algorithm needs to consist of random displacements of the centers-of-mass of the mesogens and their random rotation. Both substeps are attempted with equal probability where the specific mesogen is picked sequentially. The $N$ attempts to either displace or rotate a mesogen are followed by one attempt to change the area $A$. This is effected by rescaling the side lengths of the computational cell homogeneously. Whereas this is uncritical for states in the isotropic phase some caution is required as far as the nematic (or even smectic phases) are concerned where mesogens are predominantly aligned with $\widehat{\boldsymbol{n}}(\boldsymbol{r})$. If the simulation cell is scaled homogeneously these states could suffer from some artificial stress at least in principle. However, one anticipates this problem to be less relevant for the present model liquid crystal consisting of mesogens that are nearly spherical in shape. Nevertheless, to make sure that homogeneous scaling does not pose any problems for the present set of simulations we monitor individual components of $\mathbf{P}$ during the course of the simulation. In our simulations the computed value of $P_{\|}$ agrees with the input value to within less than $0.1 \%$.

For convenience we express all quantities in the usual customary dimensionless (i.e., "reduced") units. For example, length is given in units of $\sigma$, energy in units of $\epsilon_{\mathrm{ff}}$, and temperature in units of $\epsilon_{\mathrm{ff}} / k_{\mathrm{B}}$. Other derived quantities are expressed in terms of suitable combinations of these basic quantities. An example of such a derived quantity is the pressure which is given here in units of $\epsilon_{\mathrm{ff}} / \sigma^{3}$.

Together the $N$ rotation and translation attempts and the one attempt to change the $z$-directed area of the computation cell constitute a MC cycle. Our runs are typically based on $10^{4}$ 
equilibration cycles followed by $1-5 \times 10^{5}$ production cycles during which relevant ensemble averages are computed. Our systems comprise $N=12000$ liquid-crystal molecules and are carried out at $P_{\|}=1.80$ and for temperatures in the range $0.90 \leq T \leq 1.00$. Under these conditions the liquid crystal is always in the nematic phase with a mean number density of about 0.85 . We start our simulations from random configurations and let specific defect topologies develop during the initial equilibration period. As far as details of the model are concerned we consider two strengths $\alpha=0.40$ and 0.60 of the perpendicular anchoring function [see Eq. (2.11)]. In computing local quantities such as $\rho(x, z)$ or $\widehat{\boldsymbol{n}}(x, z)$ we discretized the $x-z$ plane into bins. Using bin sizes of about $0.2 \sigma \times 0.2 \sigma$ we made sure that per bin we had always a minimum of more than 5000 entries.

To save computer time as much as possible and because the fluid-fluid interactions are short range we employ a potential cutoff $r_{\mathrm{c}}=3.00$ at which we truncate the intermolecular interactions. No corrections are applied for neglected interactions beyond $r_{\mathrm{c}}$. Moreover, $u_{\mathrm{ff}}$ remains unshifted. However, because we vary the range of the colloid-fluid interaction potential we choose a larger cutoff radius $r_{\mathrm{c}}=5.00+r_{0}$ to make sure that $u_{\mathrm{fc}}$ has decayed to sufficiently small values such that neglecting interactions beyond $r_{\mathrm{c}}$ does not cause any spurious effects. For the fluid-fluid interactions we utilize a combination of a link-cell and a conventional Verlet neighbor list as described in the book by Allen and Tildesley ${ }^{48}$ to further speed up the simulations. This latter list includes as neighbors all mesogens whose centers-of-mass are separated by a distance $r_{\mathrm{n}}=3.5$ from that of a reference mesogen. No neighbor list is employed for the interaction between the colloid and the mesogens. For this setup a typical MC simulation took 10-12 h of central processing unit time on an AMD Opteron Quadcore 3.1 GHz processor.

\section{B. Chemically homogeneous colloids}

For reasons of comparison with previously published work $^{29,30}$ we begin with a discussion of defect structures forming in the vicinity of a homogeneous colloid. Cartoons of the most commonly observed defect topologies are presented in Fig. 3. Defect topologies of the sort depicted in Fig. 3 have been observed experimentally for colloidal particles of less than $10 \mathrm{~nm}$ in diameter ${ }^{8}$ which is the size range to which our current model systems pertain. If the anchoring function $g\left(\widehat{\boldsymbol{u}}_{i}\right)=1$ our molecules tend to assume an orientation that is perpendicular to the surface of the colloid as illustrated by the dashes in Fig. 4. As one can see from Fig. $4, \widehat{\boldsymbol{n}}(x, z)$ is parallel with the $x$-axis sufficiently far away from the surface of the colloid. The local nematic order is rather high $\left[\lambda_{+}(x, z) \simeq 0.70\right]$ almost everywhere except for relatively small areas located on the equator of the colloid. Here, $\lambda_{+}(x, z) \lesssim 0.15$. As one can see from the plot the decrease of $\lambda_{+}(x, z)$ is caused by frustration of $\widehat{\boldsymbol{n}}(x, z)$. Frustration arises because the local orientation of $\widehat{\boldsymbol{n}}(x, z)$ caused by the anchoring along the equator of the colloid is orthogonal to $\widehat{\boldsymbol{n}}_{0}$. As one realizes by comparison between Fig. 4 and the cartoon presented in Fig. 3(b) the topology of the present defect structure is that of a surface ring. (a)

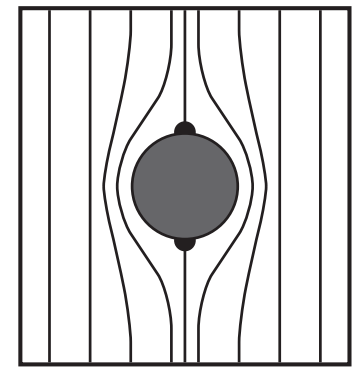

(c)

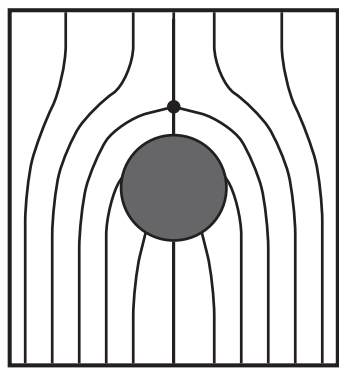

(b)

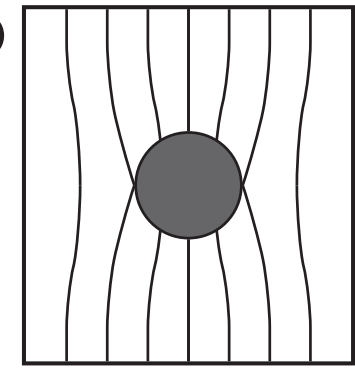

(d)

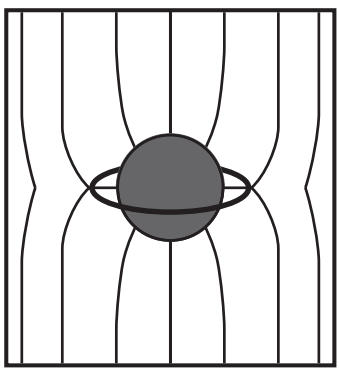

FIG. 3. Cartoons of characteristic defect structures forming in the vicinity of homogeneous spherical colloids. Solid lines indicate the direction of the local nematic director $\widehat{\boldsymbol{n}}(\boldsymbol{r})$. Defects are indicated by black dots or a solid circular line surrounding the colloid. (a) Boojum defect, (b) surface ring, (c) hedgehog, (d) saturn ring.

Next we consider the case of strong perpendicular anchoring. Figure 5 illustrates both the amount of local order and variations in the director field. The main difference between the plots in Figs. 4 and 5 is that in the latter case the region of low nematic order is moved further away from the colloid but stays at its equator otherwise. This can easily be explained because if the perpendicular anchoring at the colloid becomes stronger the region over which $\widehat{\boldsymbol{n}}(x, z)$ is determined by the colloid increases. Hence, the competition between the anchoring and $\widehat{\boldsymbol{n}}_{0}$ arises further away from the colloid's surface. Hence, from a topological perspective the

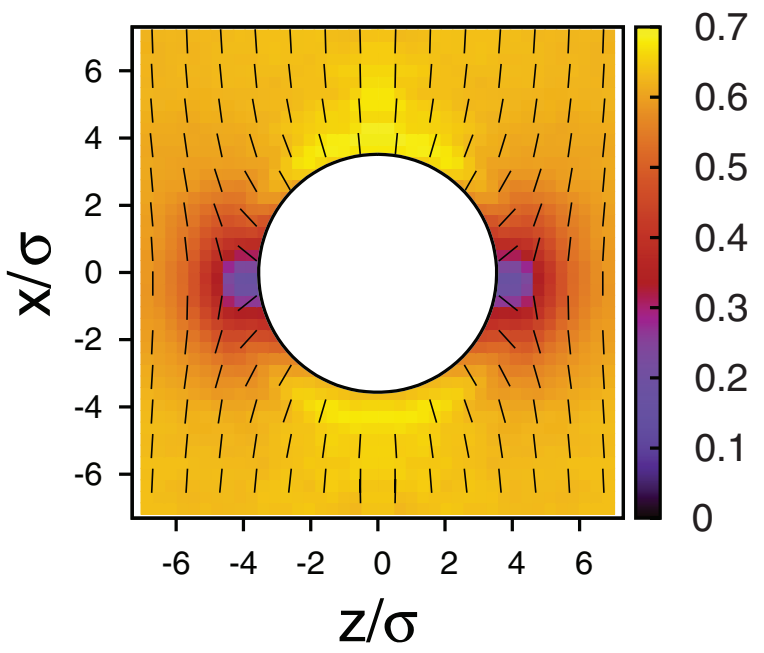

FIG. 4. Plot of the local nematic order parameter $\lambda_{+}(x, z)$ in the $x-z$ plane where the color indicates the magnitude of $\lambda_{+}(x, z)$ (see attached color bar). The white area at the center represents the homogeneous spherical colloid. Also shown is the orientation of the local nematic director $\widehat{\boldsymbol{n}}(x, z)(-)$. Data are obtained for weak perpendicular anchoring $\left(\epsilon_{\mathrm{fc}}=1.0\right.$, see text) [see Eq. (2.7)]. The solid black circle surrounding the central white area corresponds to the hard-core radius $r_{0}=3$ of the colloid. 


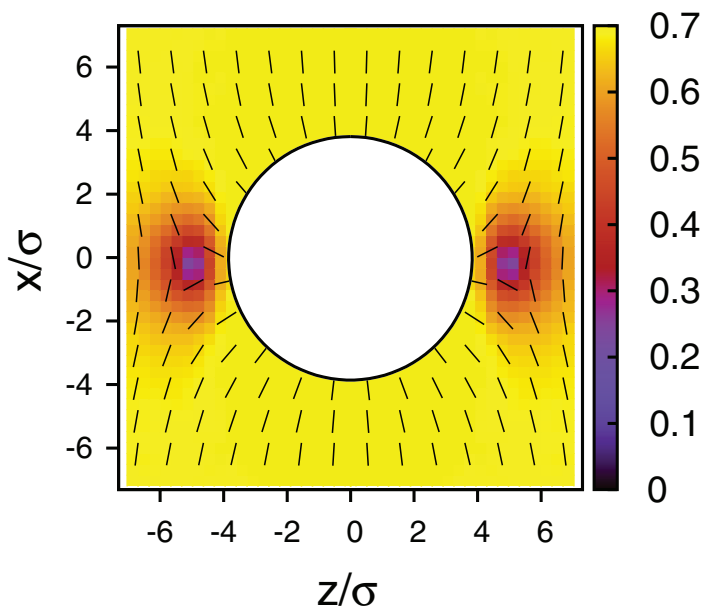

FIG. 5. As Fig. 4 but for strong perpendicular anchoring realized by setting $\epsilon_{\mathrm{fc}}=1.0, \alpha=1.00, \delta=0.00, \gamma_{\|}=1.00$, and $\gamma_{\perp}=0.00$ [see Eqs. (2.11)-(2.13)].

structures shown in Figs. 4 and 5 may be considered equivalent. Experimentally, ring defect topologies are perhaps the ones most commonly encountered. ${ }^{3,10,14}$

Saturn-ring defect topologies have first been predicted by Terentjev ${ }^{49}$ and have later been reported also by Andrienko et $a ._{.}{ }^{29}$ and by Grollau et al. ${ }^{30}$ However, in these works no specific anchoring at the colloid's surface has been employed. Consequently, a displacement of the ring defect relative to the surface of the colloid could not be observed which is solely a consequence of the strength of perpendicular anchoring that has not been varied in these earlier studies.

Andrienko et al. also reported a hedgehog defect topology schematically shown in Fig. 3(c). They observe this defect topology only for larger colloids and if the initial configuration is prepared in accordance with the hedgehog defect topology. Consequently, Andrienko et al. conclude that the hedgehog defect topology is only metastable at best. The hedgehog defect topology has also been seen experimentally $3,10,14$ and theoretically by minimizing the Frank free-energy. ${ }^{9}$

We failed to observe the hedgehog defect topology in our simulations presumably because our colloid is smaller and, more importantly, because our simulations are not starting from an initial configuration set up according to any specific defect topology. Moreover, we cannot rule out the possibility that confinement destabilizes the hedgehog defect despite our relatively large substrate separation of $s_{z}=24$. That confinement may cause a change from dipolar to quadrupolar defect topologies which has been shown experimentally by Škarabot et al. who investigated silica spheres of diameters of $2.32 \mu \mathrm{m}$ and $4.7 \mu \mathrm{m}$ immersed in 5CB as the nematic liquid crystal host phase. Bringing this system into a wedge-type nematic cell they observe dipolar defect structures in thicker parts of the cell and quadrupolar ones in narrower parts. Given the size of the silica particles used in this study and a wedge width that varied between 2 and $8 \mu \mathrm{m}$ the degree of confinement is about the same as in this work. Therefore, it may well be that in addition to a too small colloidal particle our confinement conditions prevent us from observing a hedgehog defect topology.

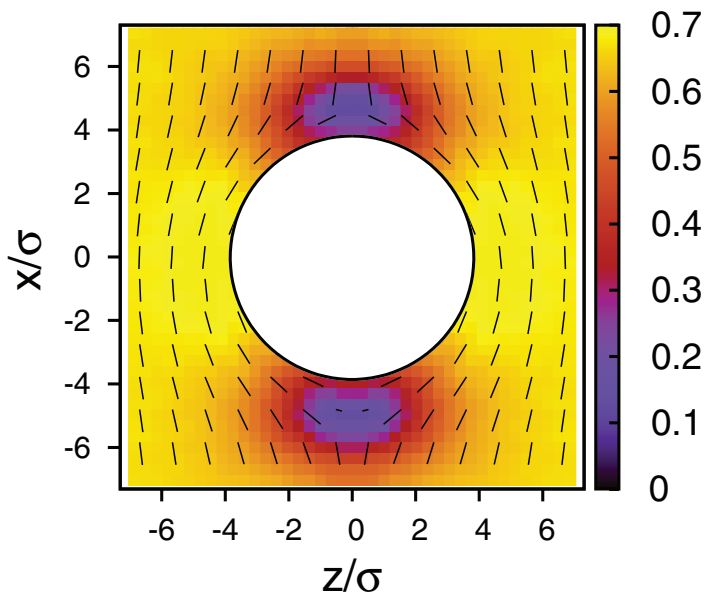

FIG. 6. As Fig. 4 but for strong parallel anchoring realized by setting $\alpha$ $=0.00, \delta=0.00, \gamma_{\|}=0.00, \gamma_{\perp}=0.00$ [see Eqs. (2.11)-(2.13)], $\epsilon_{\mathrm{fc}}$ $=4.0$, and $\eta=1.0$ [see Eq. (2.7)].

However, we succeeded in observing another defect topology that to the best of our knowledge has not yet been reported from molecular simulation. This topology is known as boojum defect schematically illustrated by the cartoon in Fig. 3(a). As one can see from Fig. 6 this defect topology arises if molecules are anchored in a locally parallel fashion at the surface of the colloid. Now portions of the liquid crystal close to the equator (i.e., around $x=0$ ) comply with $\widehat{\boldsymbol{n}}_{0}$ dictated by the solid substrates. However, portions of $\widehat{\boldsymbol{n}}(x, z)$ around north and south pole of the colloid (i.e., around $z=0$ ) and sufficiently close to its surface are more or less orthogonal to $\widehat{\boldsymbol{n}}_{0}$. The boojum defect topology has also been found experimentally. ${ }^{9}$

\section{Patchy colloids}

The results presented in Sec. IV B mainly serve to validate our model liquid crystal by demonstrating that we can reproduce known defect topologies. In addition, our approach is also capable of representing the so-called boojum defect which has been seen experimentally but apparently not in molecular simulation to date. We now turn to the focal point of our study, namely, defect topologies in the vicinity of a chemically heterogeneous, patchy colloid. As in Sec. IV B it seems helpful to analyze the defect topologies with the help of cartoons shown in Fig. 7 similar to the ones presented in Fig. 3. As one would anticipate the patchiness of the colloid causes much richer defect topologies.

We begin our discussion with Fig. 8 where we present $\widehat{\boldsymbol{n}}(\boldsymbol{r})$ and $\lambda_{+}(\boldsymbol{r})$ for the boojum ring topology schematically depicted in Fig. 7(a). As one can see from Fig. 8, the perturbation of $\widehat{\boldsymbol{n}}(\boldsymbol{r})$ is largest at the upper hemisphere where parallel anchoring dominates. The upper hemisphere is capped by a region of relatively low nematic order where molecules are oriented parallel with respect to the colloid's surface. Near the lower hemisphere, where molecules are anchored perpendicularly, nematic order is much higher as the plot in Fig. 8 reveals. The boojum ring defect topology has already 
(a)

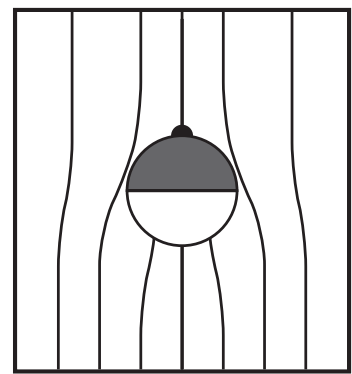

(c)

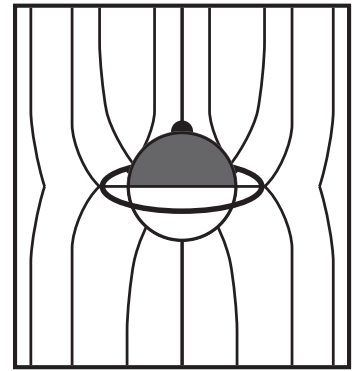

(b)

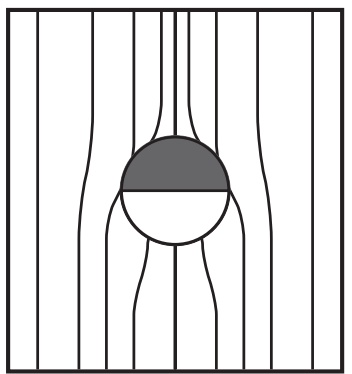

(d)

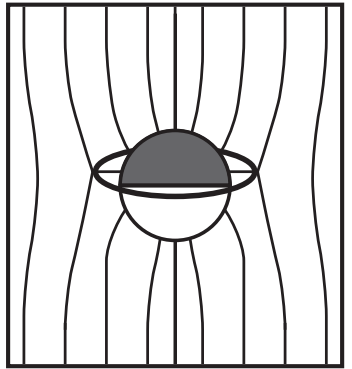

FIG. 7. As Fig. 3, but for a patchy colloid. (a) Boojum ring, (b) crown, (c) cap, and (d) off-center saturn ring.

been observed experimentally ${ }^{20,21}$ and theoretically within the framework of Landau-de Gennes mean-field theory. ${ }^{20}$

If one lowers the temperature to $T=0.90$ while maintaining all model parameters the boojum ring topology depicted in Fig. 8(a) gives way to a new "crown" topology shown in Fig. 8(b). It consists of a somewhat more extended region of ring defects centered at the north pole of the colloid where parallel anchoring is dominating according to the present choice of model parameters. However, as one can see by comparison between the plots in Figs. 8(a) and 8(b) directly at the north pole of the colloid, where parallel anchoring should be most pronounced, the orientation of the mesogens that complies with $\widehat{\boldsymbol{n}}_{0}$ is the one that prevails.

This somewhat surprising result can be rationalized as follows. Because the crown topology forms at a lower $T$ $=0.90$ compared with the boojum ring structure at $T=0.96$ the overall nematic order is higher everywhere in the liquid crystal. Hence, the free energy is lower if larger regions in the liquid-crystal carrier fluid exist for which $\widehat{\boldsymbol{n}}(x, z) \simeq \widehat{\boldsymbol{n}}_{0}$. In other words, if the overall degree of nematic order increases as $T$ is lowered perpendicular anchoring will eventually "win" especially in those regions along the colloids surface where perpendicular anchoring coincides with $\widehat{\boldsymbol{n}}_{0}$ which happens to be the case close to the north and south poles of the colloid. This causes a ring of defects to form which encircles the north pole like a "crown."

In principle, one may envision a similar formation of a "crown" defect topology starting from the boojum defect topology at a chemically homogeneous colloidal particle by lowering $T$ (see Fig. 6). However, all attempts to observe a similar "crown" topology in the case of chemically homogeneous colloids in our simulations have failed. This failure may be rationalized as follows. The boojum defect topology illustrated by the plot in Fig. 6 has been generated by setting $\alpha=0$. From Eqs. (2.7) and (2.11) it is clear that this causes a strong energetic penalty for homeotropic anchoring at the surface of

(a)

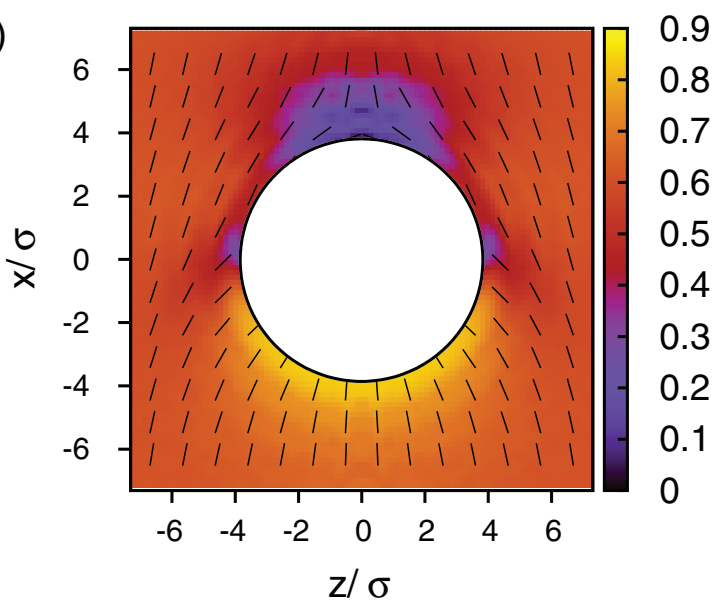

(b)

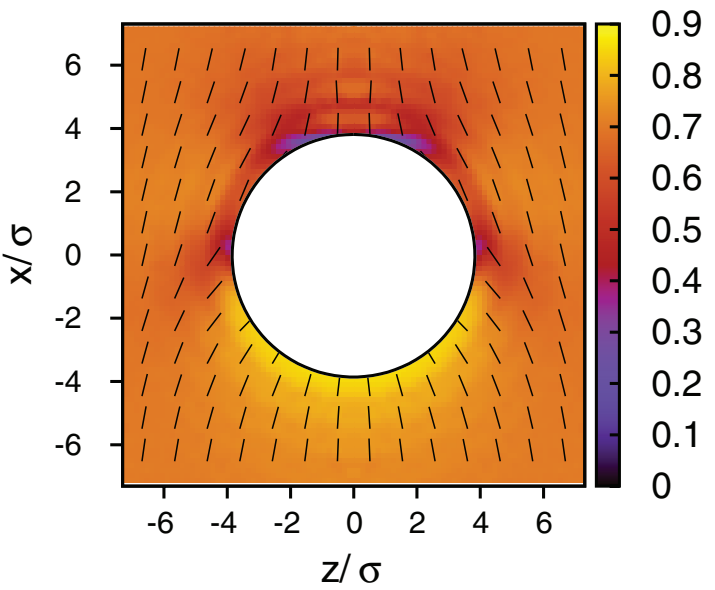

FIG. 8. As Fig. 4, but for a patchy colloid where $\alpha=0.60, \eta=0.50$, $\epsilon_{\mathrm{fc}}=4.00, \gamma=0.25, \gamma_{\perp}=0.05, \delta=2.00$ such that molecules are predominantly anchored parallel at the upper $(x>0)$ and perpendicular at the lower hemisphere $(x<0)$. (a) Boojum ring topology, $T=0.96$ [see Fig. 7(a)]; (b) crown topology, $T=0.90$ [see Fig. 7(b)].

the colloid irrespective of $T$. The defect topologies illustrated by the plots in Fig. 8, on the other hand, have been generated such that the anchoring at the upper hemisphere is predominantly planar but the homeotropic anchoring receives a less severe energetic punishment. Therefore, as $T$ is lowered the likelihood of homeotropic alignment in the direction of $\widehat{\boldsymbol{n}}_{0}$ is nonzero directly at the colloid's north pole and may eventually win over parallel alignment imposed at the surface of the colloid. However, slightly off the north pole the stronger parallel alignment prevails such that the "crown" topology becomes stable as $T$ is lowered. This is because closer to the equator of the colloid the deviation between $\widehat{\boldsymbol{n}}(\boldsymbol{r})$ and $\widehat{\boldsymbol{n}}_{0}$ is less pronounced than directly at the north pole (see Fig. 6).

A second ring of defects forms around the colloid's equator as the plots in Figs. 8(a) and 8(b) reveal. This ring is reminiscent of a surface-ring defect topology as one can see by comparison with Fig. 5 where, however, this defect ring is much more pronounced on account of the stronger perpendicular anchoring. Notice also another difference between a "true" surface ring [see Fig. 4] and the one characteristic of the crown and boojum ring defect topology. For the latter two 
(a)

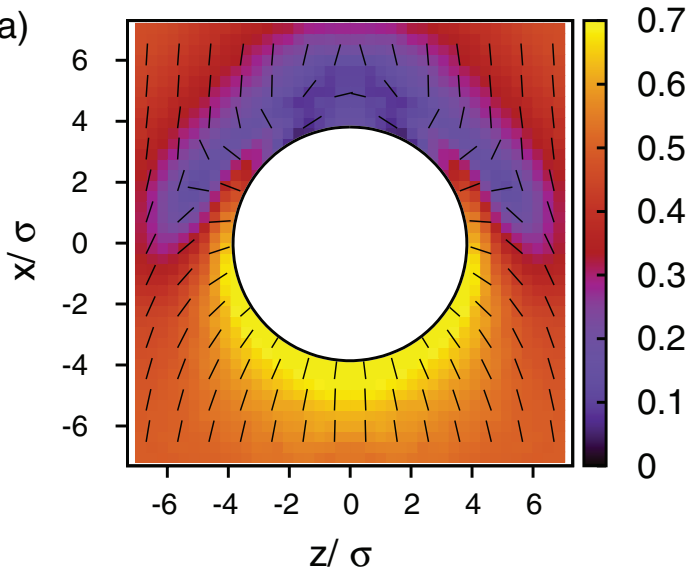

(b)

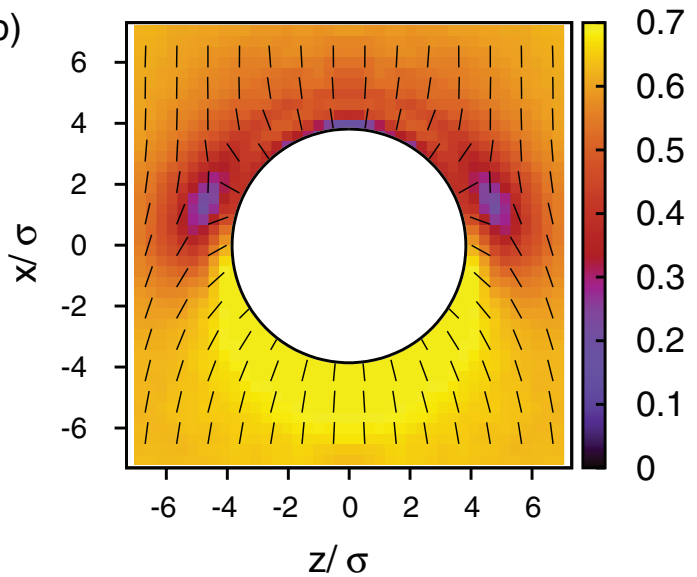

FIG. 9. As Fig. 8, but for $\alpha=0.40, \epsilon_{\mathrm{fc}}=4.00, \gamma_{\|}=0.70, \gamma_{\perp}=0.05$, and $\delta$ $=0.50$ such that molecules are predominantly anchored parallel at the upper $(x>0)$ and perpendicular at the lower hemisphere $(x<0)$. (a) Cap topology, $T=1.00$ [see Fig. 7(c)]; (b) off-center saturn topology, $T=0.95$ [see Fig. 7(d)].

the ring defect near the equator of the colloid is located in a region where perpendicular anchoring changes to planar anchoring. This ring defect arises when perpendicular anchored mesogens change their orientation to match $\widehat{\boldsymbol{n}}_{0}$ pointing along the $x$-axis, whereas for a surface ring topology the overall weak anchoring near the equator causes molecules to change their orientation to comply with $\widehat{\boldsymbol{n}}_{0}$.

To date the crown topology has not been observed either experimentally or in theory. It is also worth noting that the transformation from a boojum ring to a crown topology seems to be continuous as we have verified by reducing the temperature in small steps $\Delta T=0.01$ starting at $T=0.96$. Moreover, we could not observe any hysteresis in the transformation which indicates that the change in defect topology does not have the status of a first-order phase transition. For temperatures $T \geq 0.96$ and $T \leq 0.90$ the respective boojum ring and crown topologies seem rather stable with only minor qualitative changes as we have verified in additional simulations.

Similar effects arise if one increases the strength of planar relative to perpendicular anchoring [by reducing $\alpha$, see Eq. (2.11)] as well as size and sharpness of the transition from planar to perpendicular anchoring surface regions [by varying

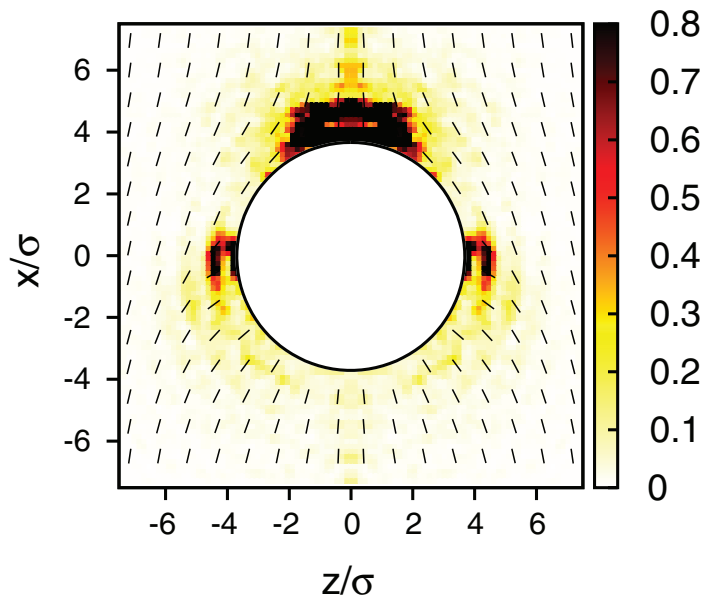

FIG. 10. Contour plot of the Frank free-energy density $\mathcal{F} / K$ in the $x-z$ plane for a boojum ring configuration at $T=0.96$ [see also Fig. 8(a)].

$\gamma_{\|}$and $\delta$, see Eq. (2.13a)]. However, the form of the defect structures forming under these conditions is distinct from the ones discussed above. This is illustrated by the plot in Fig. 9(a) where the area of low nematic order forms sort of a "cap" above the colloid's northern hemisphere. As one might have anticipated the defect near the north pole of the colloid occurs because the planar orientation of the local director changes eventually assuming the direction of $\widehat{\boldsymbol{n}}_{0}$ as $x$ increases while $z$ remains constant. Near the equator of the colloid the defect topology arises when the locally perpendicular director near the surfaces changes to the orientation of $\widehat{\boldsymbol{n}}_{0}$ as $z$ increases along lines of constant $x$. To the best of our knowledge this cap defect topology has not been reported elsewhere to date.

If, on the other hand, the temperature is reduced to $T$ $=0.95$ the plot in Fig. 9(b) illustrates the formation of a more conventional off-center saturn-ring topology which has already been seen experimentally (see Fig. 5 of Ref. 20). The off-center position of the ring defect is caused by the smaller parallel anchoring area on the colloid's surface. Again, because at $T=0.95$ the nematic order is higher than that at $T=1.00$ considered before the local nematic director near the north pole tends to coincide with $\widehat{\boldsymbol{n}}_{0}$ thereby destroying the defect region near the north pole that is clearly visible in the plot presented in Fig. 9(a).

\section{Analysis of the Frank free-energy density}

To gain more detailed insight into the specific deformation of the director field we now turn to a discussion of the Frank free-energy density introduced in Eq. (3.17). In Fig. 10 we show a plot of the local Frank free-energy density for the boojum ring configuration together with the topology of the director field. A comparison between the plots in Figs. 8(a) and 10 reveals that $\mathcal{F}$ is largest in regions where the local nematic order parameter is lowest according to one's expectation. The question then arises: which contribution to $\mathcal{F}$ determines its total value? To address this question we display in Fig. 11 plots of splay, bend, and twist contributions to $\mathcal{F}$. 
(a)

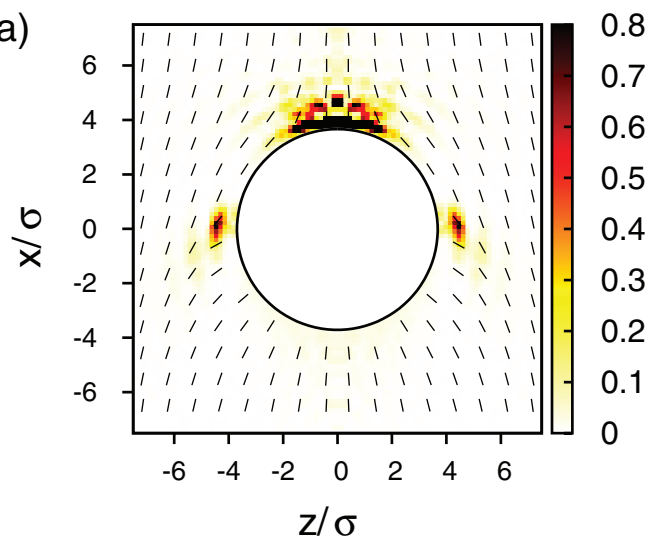

(b)

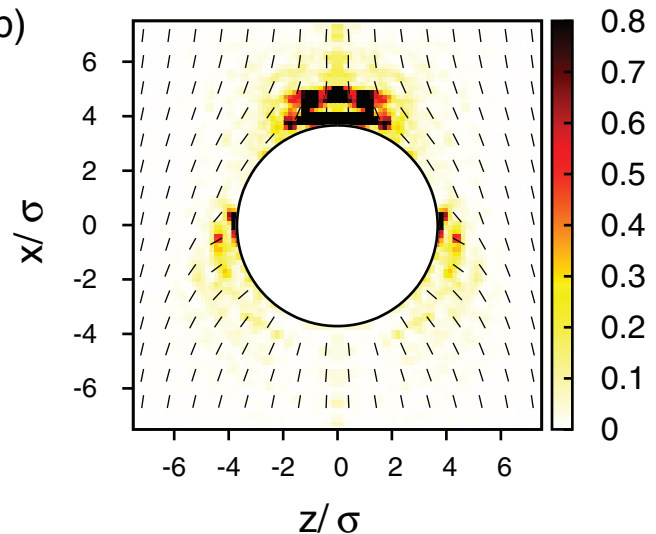

(c)

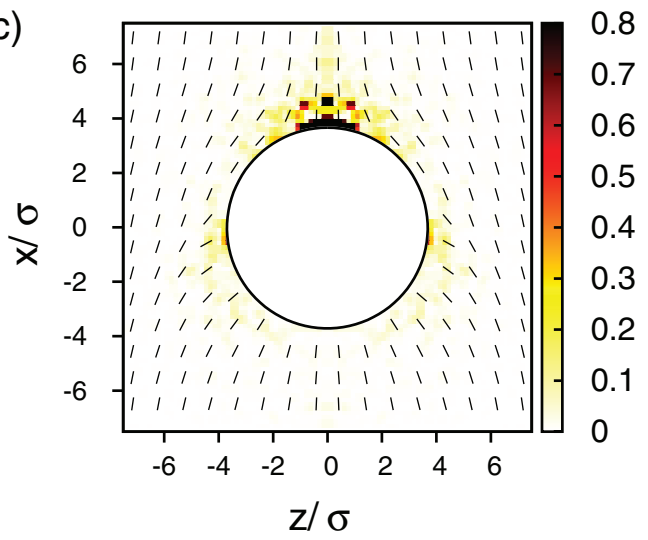

FIG. 11. As Fig. 10, but for splay (a), bend (b), and (c) twist contributions [see Eq. (3.17)].

Plots in Fig. 11 clearly show that the largest contributions to $\mathcal{F}$ are due to splay and bend deformations of $\widehat{\boldsymbol{n}}(\boldsymbol{r})$ where the bend contributions appear to be somewhat more spatially extended. This seems to make some physical sense because both the boojum and the ring defect topologies are located in an area where the liquid crystal molecules are anchored at the surface of the colloid such that the angle between $\widehat{\boldsymbol{n}}(\boldsymbol{r})$ and the far-field director $\widehat{\boldsymbol{n}}_{0}$ is largest and hence the local deformation of the director field is most pronounced.

The plot in Fig. 11(c), on the other hand, reveals that there is only a very small contribution of the twist contribution to $\mathcal{F}$ according to one's physical intuition. This is because the twist configuration [see Fig. 3(c)] comes about only if $\widehat{\boldsymbol{n}}(\boldsymbol{r})$ rotates in a plane orthogonal to a local surface normal to the colloid and as the distance from the colloid's surface increases [see Fig. 3(c)]. As the plot in Fig. 11(c) reveals significant twist contributions are limited to a relatively small region centered on the colloid's north pole.

\section{SUMMARY AND CONCLUSIONS}

In this work we employ MC simulations in the isothermal-isobaric ensemble to investigate the perturbation and defect topology of the local director field $\widehat{\boldsymbol{n}}(\boldsymbol{r})$ of a nematic liquid crystal due to the presence of a spherical colloidal particle. We model the liquid-crystal phase via the Hess-Su potential which treats mesogens as Lennard-Jones spheres with an orientation-dependent attractive contribution. ${ }^{22}$ Even though in other liquid-crystal models ordered (nematic or smectic) phases are usually controlled by the anisotropic shape of the molecules, the Hess-Su model has been repeatedly demonstrated to represent the characteristic mesophases of a typical thermotropic liquid crystal sufficiently realistically. ${ }^{23-28}$

The far-field director $\widehat{\boldsymbol{n}}_{0}$ is determined by the anchoring of the liquid-crystal mesogens at the surface of two structureless, planar solid substrates. These substrates only serve as a technical means to fix $\widehat{\boldsymbol{n}}_{0}$ in space but have no other physical significance. Hence, deviations between $\widehat{\boldsymbol{n}}_{0}$ and $\widehat{\boldsymbol{n}}(\boldsymbol{r})$ arise only on account of

1. the curvature of the colloid and

2. the chemical decoration of the colloid's surfaces by two patches of variable size.

The patches are realized such that mesogens are anchored at the surface of the colloid either in a predominantly planar or perpendicular fashion. These anchoring conditions compete with $\widehat{\boldsymbol{n}}_{0}$ such that $\widehat{\boldsymbol{n}}(\boldsymbol{r})$ is perturbed locally in the vicinity of the colloidal particle.

Here we have concentrated only on one particular size of the colloid because of the already huge parameter space of our model system. However, it would clearly be interesting to investigate the robustness of the defect topologies observed in this study against a variation of the size of the colloidal particle. A particularly interesting case in this regard might be to go to smaller colloids and even to the limit in which the colloid becomes comparable in size to a mesogen where curvature effects become irrelevant.

Depending on the specific anchoring conditions different defect topologies arise. Taking homogeneous colloids as a reference system we demonstrate that not only does our model reproduce already known topologies such as surface ring and saturn ring but also accounts for a boojum defect topology in the case of strong, homogeneous parallel anchoring at the surface of the colloidal particle. To the best of our knowledge this study seems to be the first one in which the boojum topology has been observed in molecular simulations.

For a heterogeneous, patchy colloid the defect topology is generally richer. For example, a boojum ring topology is observed which may be perceived as a combination of a boojum defect topology and a surface-ring topology both of which may be realized with a chemically homogeneous colloid. That 
this topology is a realistic one has very recently been demonstrated experimentally ${ }^{20,21}$ which further validates the modeling approach on which our study is based. However, some caution may be advisable when comparing experimental and theoretical data for colloids that mismatch strongly in size. In this latter case an agreement between both data sets could be fortuitous.

However, our results also predict several new defect topologies that have not yet been observed either experimentally or theoretically. One of these is the so-called "crown topology." It deviates from the boojum ring topology in that at the colloid's north pole, where molecules are anchored in a perpendicular fashion, a region of relatively high nematic order exists that is surrounded by a region of lower nematic order. Over a small range of temperatures the boojum ring and the crown topologies may be transformed into one another reversibly without any hysteresis. Thus, it seems likely that this temperature-induced transformation should not be considered to be a surface phase transition. Above and below this temperature range both boojum ring and crown topologies remain stable and undergo only minute changes if the temperature is not raised or lowered extremely.

If one increases the strength of parallel anchoring relative to the perpendicular one the "cap topology" evolves. It is characterized by a region of low nematic order that covers the northern hemisphere of the colloid like a cap. The "cap topology" causes the most long-range perturbation of the director field of all the defect topologies that are reported in this work. Finally, if one lowers the temperature the "cap topology" is transformed into a more conventional off-center saturn ring topology that has already been observed experimentally. ${ }^{20}$

The various defect topologies investigated here manifest themselves also in the topology of the local Frank free-energy density $\mathcal{F}$ in that regions of lower nematic order give rise to an increase in $\mathcal{F}$. This increase is largely due to splay and bend deformations of $\widehat{\boldsymbol{n}}$ in all the cases investigated. However, to draw any quantitative conclusions about the relative importance of twist, splay, and bend contributions to $\mathcal{F}$ would require to abandon the one-constant approximation and to determine all three elastic constants separately. This would be possible in a computer simulation employing an approach successfully used by Allen and co-workers some time ago. ${ }^{50}$ Their data obtained for elongated Gay-Berne molecules show that the three elastic constants are not identically the same (as in the one-constant approximation) but are roughly of the same order of magnitude. Hence, our current qualitative analysis of $\mathcal{F}$ may actually be not too bad particularly in view of the nearly spherical shape of our mesogens. More recently, Phuong et al. calculated all three elastic constants from direct correlation functions and from the long-range fluctuations of the alignment tensor in Fourier space. ${ }^{51}$

Given the complexity of defect topologies it would also be interesting to study how the topologies of a pair of colloids interfere with each other depending on their relative orientation and distance from one another. Thinking about $\mathcal{F}$ as an effective (i.e., state-dependent) potential acting between a pair of colloids it then seems conceivable that different defect topologies may cause a distinctly different way in which (homogeneous and patchy) colloids may self-assemble to larger supramolecular structures with rather different material properties. By controlling the self-assembly through changes in defect topology induced by external agents such as temperature one may be able to tailor self-assembled nanostructures or microstructures with specific and desired properties. In this sense the composite colloid/liquid crystal systems considered here seem to be versatile candidates to study complex selfassembly as are colloidal systems with "naturally" anisotropic interactions such as dipolar particles. ${ }^{52}$

However, even though the local Frank free-energy density may in principle offer a route to study self-assembly in a dispersion of colloidal particles in a coarse-grained fashion without having to consider the liquid-crystal carrier phase $e x$ plicitly, the present study clearly highlights the importance of molecule-based, microscopic approaches. This is because the rather complex defect topologies have been shown to arise as a consequence of a rather complex interplay between various molecular features of our model system.

\section{ACKNOWLEDGMENTS}

Financial support from the International Graduate Research Training Group 1524 "Self-assembled soft matter nanostructures at interfaces" is gratefully acknowledged.

${ }^{1}$ H. Stark, Phys. Rep. 351, 387 (2001).

${ }^{2}$ S. H. L. Klapp, S. Grandner, Y. Zeng, and R. von Klitzing, Soft Matter 6, 2330 (2010).

${ }^{3}$ U. Ognysta, A. Nych, V. Nazarenko, M. Škarabot, and I. Muševič, Langmuir 25, 12092 (2009).

${ }^{4}$ S. Grandner and S. H. L. Klapp, Europhys. Lett. 90, 68004 (2010).

${ }^{5}$ J. Joannopoulos, S. Johnson, and J. Winn, Photonic Crystals: Molding the Flow of Light (Princeton University Press, Princeton, 2008).

${ }^{6}$ E. Yablonovitch, Phys. Rev. Lett. 58, 2059 (1987).

${ }^{7}$ D. Grier, MRS Bull. 23, 21 (1998).

${ }^{8}$ H. Qi and T. Hegmann, J. Mater. Chem. 16, 4197 (2006).

${ }^{9}$ P. Poulin, H. Stark, T. C. Lubensky, and D. A. Weitz, Science 275, 1770 (1997).

${ }^{10}$ P. Poulin and D. A. Weitz, Phys. Rev. E 57, 626 (1998).

${ }^{11}$ J.-C. Loudet, P. Barois, and P. Poulin, Nature (London) 407, 611 (2000).

${ }^{12}$ A. A. Sonin, The Surface Physics of Liquid Crystals (Gordon and Breach, Amsterdam, 1995).

${ }^{13}$ A. Figuerola, A. Fiore, R. D. Corato, A. Falqui, C. Giannini, E. Micotti, A. Lascialfari, M. Corti, R. Cingolani, T. Pellegrino, P. D. Cozzoli, and L. Manna, J. Am. Chem. Soc. 130, 1477 (2008).

${ }^{14}$ M. Škarabot, M. Ravnik, S. Žumer, U. Tkalec, I. Poberaj, D. Babič, N. Osterman, and I. Muševič, Phys. Rev. E 77, 031705 (2008).

${ }^{15}$ O. Mondain-Monval, J. C. Dedieu, T. Gulik-Krzywicki, and P. Poulin, Eur. Phys. J. B 12, 167 (1999).

${ }^{16}$ C. Casagrande and M. Veyssié, C. R. Acad. Sci. Paris, Ser. II 306, 1423 (1988).

${ }^{17}$ A. Walther and A. H. E. Müller, Soft Matter 4, 663 (2008).

${ }^{18}$ A. Perro, S. Reculusa, S. Ravaine, E. Bourgeat-Lami, and E. Duguet, J. Mater. Chem. 15, 3745 (2012).

${ }^{19}$ X. Y. Ling, L. Y. Phang, G. Acikgoz, M. D. Yilmaz, M. A. Hempenius, G. J. Vancso, and J. Huskens, Angew. Chem. 121, 7813 (2009).

${ }^{20}$ M. Conradi, M. Ravnik, M. Bele, M. Zorko, S. Žumer, and I. Muševič, Soft Matter 5, 3905 (2009).

${ }^{21}$ M. Conradi, M. Zorko, and I. Muševič, Opt. Express 18, 500 (2010).

${ }^{22} \mathrm{~S}$. Hess and B. Su, Z. Naturforsch. 54a, 559 (1999).

${ }^{23}$ M. Greschek and M. Schoen, Phys. Rev. E 83, 011704 (2011).

${ }^{24}$ M. Greschek and M. Schoen, J. Chem. Phys. 135, 204702 (2011).

${ }^{25}$ H. Steuer, S. Hess, and M. Schoen, Physica A 328, 322 (2003).

${ }^{26} \mathrm{H}$. Steuer, S. Hess, and M. Schoen, Phys. Rev. E 69, 031708 (2004).

${ }^{27}$ M. Greschek, M. Melle, and M. Schoen, Soft Matter 6, 1898 (2010).

${ }^{28}$ M. Greschek and M. Schoen, Soft Matter 6, 4931 (2010). 
${ }^{29}$ D. Andrienko, G. Germano, and M. P. Allen, Phys. Rev. E 63, 041701 (2001).

${ }^{30}$ S. Grollau, E. B. Kim, O. Guzmán, N. L. Abbott, and J. J. de Pablo, J. Chem. Phys. 119, 2444 (2003).

${ }^{31}$ S.-H. Hu and X. Gao, J. Am. Chem. Soc. 132, 7234 (2010).

${ }^{32}$ S. Gangwal, A. Pawar, I. Kretzschmar, and O. D. Velev, Soft Matter 6, 1413 (2010).

${ }^{33}$ D. J. Diestler, M. Schoen, J. E. Curry, and J. H. Cushman, J. Chem. Phys. 100, 9140 (1994).

${ }^{34}$ M. Schoen, Physica A 270, 353 (1999).

${ }^{35} \mathrm{M}$. Schoen and S. Klapp, Nanoconfined Fluids. Soft Matter Between Two and Three Dimensions (Wiley-VCH, New York, 2007).

${ }^{36}$ C. G. Gray and K. E. Gubbins, Theory of Molecular Fluids, Vol. 1 (Clarendon, Oxford, 1984).

${ }^{37}$ T. Gruhn and M. Schoen, J. Chem. Phys. 108, 9124 (1998).

${ }^{38}$ I. Pardowitz and S. Hess, Physica A 100, 540 (1980).

${ }^{39}$ R. Eppenga and D. Frenkel, Mol. Phys. 52, 1303 (1984).

${ }^{40}$ W. Press, S. A. Teukolsky, W. T. Vetterling, and B. P. Flannery, Numerical Recipes in FORTRAN (Cambridge University Press, Cambridge, 1989), Chap. 11.1.
${ }^{41}$ C. Zannoni, The Physics of Liquid Crystals (Academic, London, 1979).

${ }^{42}$ H. Weber, W. Paul, and K. Binder, Phys. Rev. E 59, 2168 (1999).

${ }^{43}$ A. Richter and T. Gruhn, J. Chem. Phys. 125, 064908 (2006).

${ }^{44}$ P. G. de Gennes and J. Prost, The Physics of Liquid Crystals (Oxford Science Publications, Oxford, 1995), Chap. 3.

${ }^{45}$ R. W. Ruhwandl and E. M. Terentjev, Phys. Rev. E 56, 5561 (1997).

${ }^{46}$ T. C. Lubensky, D. Pettey, N. Currier, and H. Stark, Phys. Rev. E 57, 610 (1998).

${ }^{47}$ H. Stark, Eur. Phys. J. B 10, 311 (1999).

${ }^{48}$ M. P. Allen and D. J. Tildesley, Computer Simulations of Liquids (Clarendon, Oxford, 1989), Chap. 5.3.

${ }^{49}$ E. M. Terentjev, Phys. Rev. E 51, 1330 (1995).

${ }^{50}$ M. P. Allen, M. A. Warren, M. R. Wilson, A. Sauron, and W. Smith, J. Chem. Phys. 105, 2850 (1996).

${ }^{51}$ N. H. Phuong, G. Germano, and F. Schmid, J. Chem. Phys 115, 7227 (2001).

${ }^{52}$ H. Schmidle, C. K. Hall, O. D. Velev, and S. H. L. Klapp, Soft Matter 8, 1521 (2012). 